\title{
Lumboperitonealer Shunt möglicherweise wirksam
}

Fragestellung: Ist ein lumboperitonealer Shunt zur Behandlung des Normaldruckhydrocephalus wirksam?

Hintergrund: Der Normaldruckhydrocephalus geht mit einer Gangapraxie, kognitiven Störungen und Blasenfunktionsstörungen einher. Typische Veränderungen in der zerebralen Bildgebung sind erweiterte Ventrikel und verstrichene kortikale Hirnfurchen. In den meisten Kliniken wird heute eine Probepunktion des Liquors durchgeführt und bei Besserung der Symptome dann ein ventrikuloatrialer oder lumboperitonealer Shunt eingelegt. Gute randomisierte Studien zum Nutzen einer Shunt-Anlage fehlen allerdings bisher.

Patienten und Methodik: Die Studie wurde an 20 neurologischen und neurochirurgischen Zentren in Japan durchgeführt. Die Patienten im Alter zwischen 60 und 85 Jahren mussten eine typische klinische Symptomatik für einen Normaldruckhydrocephalus aufweisen und eine typische zerebrale Bildgebung in der Kernspintomografie. Sie wurden in zwei Gruppen randomisiert: Gruppe 1 wurde unmittelbar mit einem lumboperitonealen Shunt versorgt, bei Gruppe 2 erfolgte der Eingriff um drei Monate verzögert. Der primäre Endpunkt war eine Verbesserung von einem Punkt oder mehr auf der modifizierten Rankin-Skala (mRS) drei Monate nach Randomisierung. Der sekundäre Endpunkt war der Outcome nach zwölf Monaten.

Kazui H, Miyajima M, Mori E et al; and SINPHONI-2 Investigators. Lumboperitoneal shunt surgery for idiopathic normal pressure hydrocephalus (SINPHONI-2): an open-label randomised trial. Lancet Neurol 2015; 14: 585-94
Ergebnisse: In die Gruppe der Patienten, die unmittelbar operiert wurden, wurden 49 Patienten randomisiert, in die mit dem verzögerten Eingriff 44 Patienten. Die Patienten waren im Mittel 76 Jahre alt. Die Symptome bestanden seit durchschnittlich $24 \mathrm{Mo}$ -

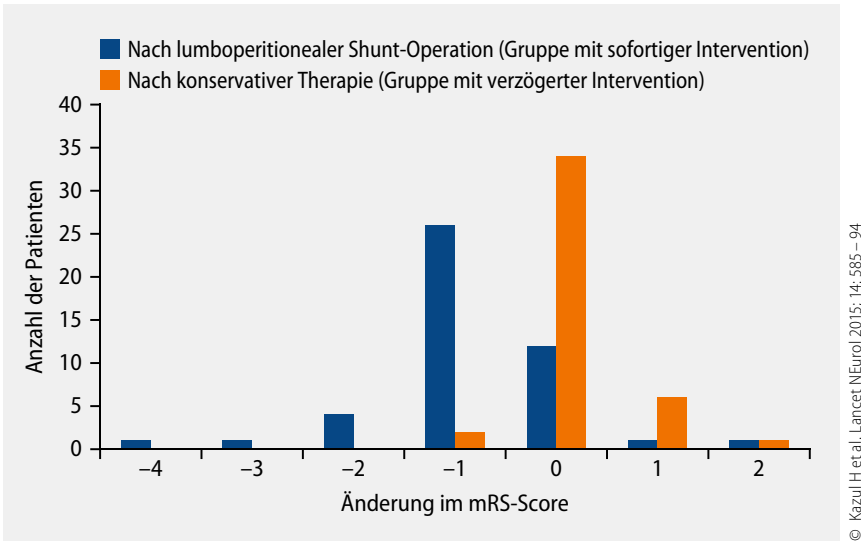

1 Änderung der mRS-Scores drei Monate nach Randomisierung.

naten. $60 \%$ der Patienten hatten die volle Symptomtrias. In der unmittelbar operierten Gruppe zeigten $65 \%$ der Patienten eine Verbesserung des $\mathrm{mRS}$-Scores, in der verzögert operierten Gruppe nur $5 \%$. Dieser Unterschied war statistisch signifikant. Abbildung 1 zeigt die Änderung im mRS-Score in den ersten drei Monaten nach Randomisierung ( Abb. 1). Beobachtete man den zwölfmonatigen Verlauf, waren 30 von 45 Patienten in der ersten Gruppe und 22 von 38 in der verzögert operierten Gruppe verbessert (67\% vs. $58 \%$, nicht signifikant). Im 12-Monats-Verlauf trat bei drei Patienten ein subdurales Hämatom auf, das operiert werden musste, bei fünf musste der Shunt revidiert werden und bei sechs Patienten trat ein Schlaganfall auf.

Schlussfolgerungen: In einer relativ kleinen offenen Studie hatte ein lumboperitonealer Shunt bei Patienten mit Normaldruckhydrocephalus eine positive Auswirkung auf die Funktionsfähigkeit im Alltag.

\section{- Kommentar von Hans-Christoph Diener, Essen}

\section{Ungeklärt bleibt, wie viele Patienten von der Operation profitieren}

Es ist immer wieder erstaunlich, dass für viele apparative Methoden und interventionelle Eingriffe in der Medizin kaum Daten aus randomisierten Studien zur Verfügung stehen. Im vorliegenden Fall wurde ein Kompromiss zwischen wissenschaftlichem Anspruch und praktischer Durchführbarkeit angestrebt, in dem eine Gruppe von Patienten mit Normaldruckhydrocephalus sofort und die andere mit einer Zeitverzögerung von drei Monaten operiert wurden.

Bei einem Eingriff, bei dem weiterhin nicht wirklich geklärt ist, wie viele Patienten letztendlich von der Operation profi- tieren, wäre es auch durchaus vertretbar gewesen, die Patienten insgesamt in eine operative und konservative Therapiegruppe zu randomisieren. Ungeachtet der relativ geringen Zahl von Patienten in der Studie, ergab sich eine signifikante Überlegenheit einer sofortigen Operation, wobei sich alle drei Symptomkomplexe des Normaldruckhydrocephalus signifikant verbesserten. Die Studie kann allerdings die Frage nicht beantworten, ob die Patienten auch von wiederholten Lumbalpunktionen mit Ablassen größerer Mengen von Liquor profitiert hätten. 